\title{
Pattern Recognition to Identify Stroke in the Cognitive Profile: Secondary Analyses of a Prospective Cohort Study
}

\author{
Sean A.P. Clouston Yun Zhang Dylan M. Smith \\ Program in Public Health and Department of Family, Population, and Preventive Medicine, \\ Stony Brook University, Stony Brook, NY, USA
}

\author{
Keywords \\ Neuroepidemiology · Cerebrovascular diseases · Pattern recognition · Adaptive diagnostics
}

\section{Abstract}

Background: Stroke can produce subtle changes in the brain that may produce symptoms that are too small to lead to a diagnosis. Noting that a lack of diagnosis may bias research estimates, the current study sought to examine the utility of pattern recognition relying on serial assessments of cognition to objectively identify stroke-like patterns of cognitive decline (pattern-detected stroke, p-stroke). Methods: Secondary data analysis was conducted using participants with no reported history of stroke in the Health and Retirement Study, a large $(n=16,113)$ epidemiological study of cognitive aging among respondents aged 50 years and older that measured episodic memory consistently biennially between 1996 and 2014. Analyses were limited to participants with at least 4 serial measures of episodic memory. Occurrence and date of $p$-stroke events were identified utilizing pattern recognition to identify stepwise declines in cognition consistent with stroke. Descriptive statistics included the percentage of the population with p-stroke, the mean change in episodic memory resulting in stroke-positive testing, and the mean time between $p$-stroke and first major diagnosed stroke. Statistical analyses comparing cases of $p$-stroke with reported major stroke relied on the area under the receiver-operating curve (AUC). Longitudinal modeling was utilized to examine rates of change in those with/without major stroke after adjusting for demographics. Results: The pattern recognition protocol identified 7,499 p-strokes that went unreported. On average, individuals with $\mathrm{p}$-stroke declined in episodic memory by $1.986(\mathrm{SD}=0.023)$ words at the in- 
ferred time of stroke. The resulting pattern recognition protocol was able to identify selfreported major stroke $(A \cup C=0.58,95 \% \mathrm{Cl}=0.57-0.59, p<0.001)$. In those with a reported major stroke, $\mathrm{p}$-stroke events were detectable on average $4.963(4.650-5.275)$ years $(p<$ $0.001)$ before diagnosis was first reported. The incidence of $p$-stroke was $40.23 / 1,000$ (95\% $\mathrm{Cl}=39.40-41.08$ ) person-years. After adjusting for sex, age was associated with the incidence of $\mathrm{p}$-stroke and major stroke at similar rates. Conclusions: This is the first study to propose utilizing pattern recognition to identify the incidence and timing of $p$-stroke. Further work is warranted examining the clinical utility of pattern recognition in identifying $p$-stroke in longitudinal cognitive profiles.

\section{Introduction}

Major stroke affects more than 800,000 Americans and causes 140,000 deaths annually, and stroke resulting from cerebrovascular or ischemic disease has potentially severe consequences [1]. Despite having a range of noticeable symptoms, including a rapid decline in cognitive function, many strokes are never diagnosed [2]. Stroke may cause reduced cognitive capacity, increased disorientation, and, ultimately, onset of cognitive impairment and dementia [2]. Notably, researchers have long noted that major strokes contemporaneously cause rapid losses in cognitive function before stabilizing [3]. However, despite having a range of noticeable symptoms, many strokes are never diagnosed [4] and, additionally, many smaller strokes may not cause obvious symptoms, as evidenced by a high prevalence of undiagnosed microinfarctions in older adults [5].

Difficulties in reliably detecting strokes may lead to delays in diagnosis, suggesting that there is a need to identify novel methods to objectively detect the onset of a new stroke. A recent study proposed to identify onset of medical conditions using longitudinal pattern recognition models [6]. While these methods are promising, especially regarding their ability to disambiguate causes of cognitive decline in fields that currently do not make a clear distinction between causes [7], to our knowledge no studies to date have utilized pattern recognition to detect a new stroke.

The current study represents an initial attempt to apply pattern recognition modeling to the identification of stroke so as to more accurately identify the timing of stroke events while also examining the extent to which such events could be identified among individuals who do or do not report an incident stroke. We hypothesized that changes in episodic memory following the stroke pattern of cognitive decline would be evident in individuals reporting major stroke. Additionally, we hypothesized that using this method would also identify possible stroke events that may not reliably produce diagnosable symptoms.

\section{Methods}

Data from waves 1-12 of the Health and Retirement Study (HRS), which collects cognitive data biennially starting in 1992 (response rate $=81.6 \%$ at baseline), were secondarily analyzed for this study. The HRS is open to enrollment at subsequent waves, and data are publicly available online (http://hrsonline.isr.umich.edu) [8]. Because of the intense analytic requirements for differential diagnostic routines, respondents without at least four waves of data were excluded. The analytic sample, therefore, included 16,113 respondents who were observed a total of 111,349 times for up to 20 years. 


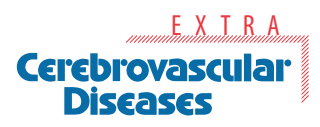

Fig. 1. Theoretically differentiating expectations in healthy aging from cognitive declines consistent with cerebrovascular and ischemic diseases. Note that results from this study may help to identify when possible cerebrovascular events are insufficient to cause dementia as noted by the lack of crossover of the cerebrovascular and ischemic disease trajectory and the clinically diagnostic threshold.

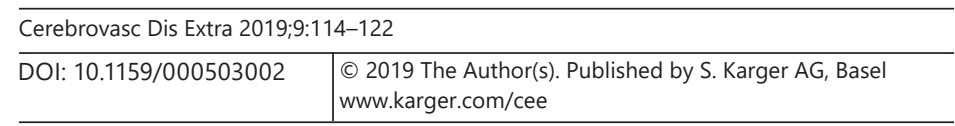

Clouston et al.: Stroke-Like Patterns of Cognitive Decline

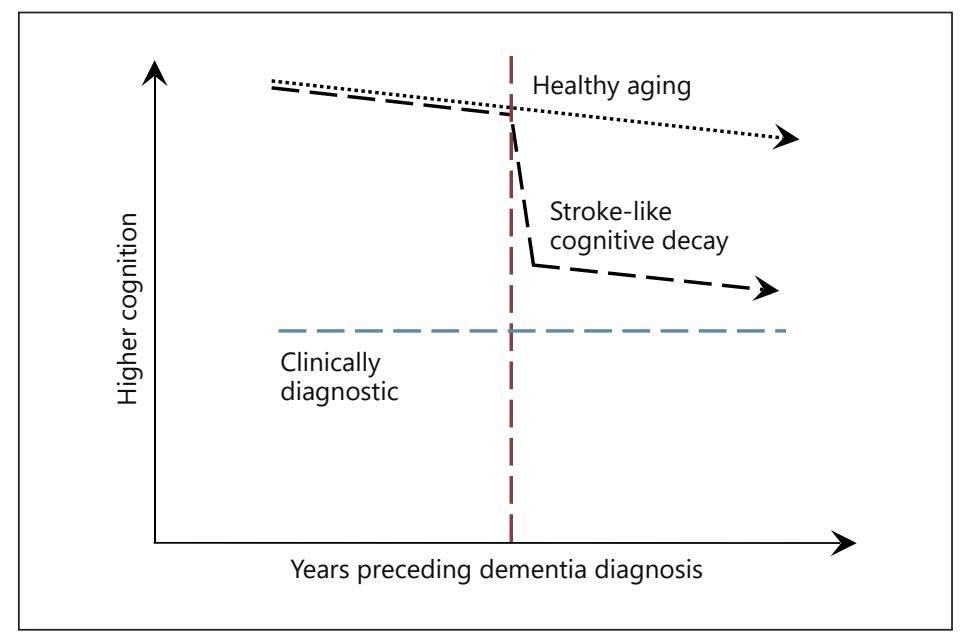

\section{Measures}

Episodic memory is a critical measure of cognitive functioning that is sensitive to cognitive aging and other dementing diseases [9]. To measure episodic memory, respondents were first provided with a list of ten words and asked to correctly recall to their best ability with each correct answer scoring one point. After approximately $10 \mathrm{~min}$ of intermediate distraction questions, respondents were asked again to repeat all ten words correctly to the interviewer to score the correct answers. The Total Episode Memory Index included the summation of both immediate and delayed verbal recall tests (/20 points). Because the first two waves utilized a 20-item word list, these waves were excluded from the current analyses.

Orientation measures the degree of confusion over everyday facts, such as the day of the week or the current president's name, that may indicate orientation in everyday life. In the HRS, orientation is measured among individuals aged 65 years and older using seven questions including the day of the week, date (month, day, year), object naming, and the name of the president and the vice president.

\section{Diagnosis of Major Stroke}

A history (and incidence) of diagnosed major stroke was recorded on intake and monitoring surveys, and by individuals and/or their proxy as necessary. This method has been shown to be highly reliable (area under the receiver-operating characteristic curve, AUC = 0.99) when identifying major stroke [10].

\section{Inferential Diagnoses}

Diagnostic categories were defined using a pattern recognition algorithm that was applied to each respondent in the database. The goal was to identify individuals whose longitudinal patterns of decline matched a stroke-like pattern of stepwise decline similar to the pattern shown in Figure 1. To accomplish this, a program sifted through individual data and determined whether each person's data best fit the profile of a linear pattern of change, or a stepwise-linear pattern consistent with stroke pattern symptoms. Since the pattern recognition program was particularly sensitive to random variation in the first or last waves, onsets that occurred before the second or after the penultimate wave were ignored. Patient group (stroke vs. no stroke) and the best-fitting date of onset were recorded.

Respondent age in years, sex, race/ethnicity, and education in years were included in multivariable analyses. Since respondents with cognitive issues sometimes misreport 

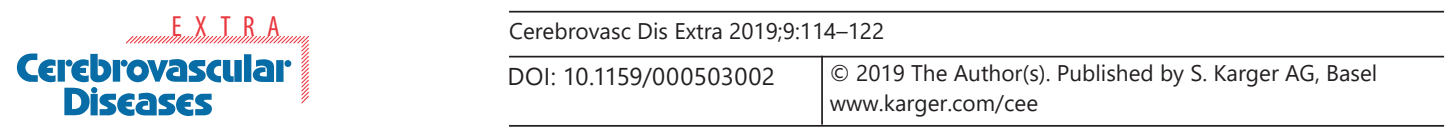

Clouston et al.: Stroke-Like Patterns of Cognitive Decline

Table 1. Sample characteristics at baseline of eligible participants for the entire sample separated by inferential diagnosis

\begin{tabular}{lccc}
\hline Characteristic & Whole sample & No p-stroke & p-stroke \\
\hline Age (mean \pm SD), years & $61.97 \pm 8.71$ & $59.72 \pm 7.33$ & $64.17 \pm 9.36$ \\
Education (mean \pm SD), years & $12.21 \pm 3.38$ & $12.43 \pm 3.24$ & $12.36 \pm 3.19$ \\
\hline Individuals, \% & & & \\
$\quad$ Race/ethnicity & & & 77.59 \\
$\quad$ White & 76.02 & 74.41 & 12.77 \\
$\quad$ Black & 13.77 & 14.79 & 7.61 \\
$\quad$ Hispanic & 1.25 & 8.90 & 2.02 \\
$\quad$ Other & 58.91 & 1.90 & 60.72 \\
Female sex & & 57.06 & \\
\hline
\end{tabular}

current age, age in years was calculated using the interview date and the date of birth. Year of birth was included in longitudinal models to capture secular change in cognitive function. Since there is a common reduction in cognitive function at the first time point due to unfamiliarity with testing circumstances, a dichotomous indicator was incorporated that identified the first wave of assessment in both longitudinal modeling and pattern recognition analyses.

\section{Statistical Analyses}

Means, standard deviations, and percentages were used to describe the sample. Crude incidence rates (IR) were stratified for age. Crude IR as well as age-standardized IR were provided. The predictive value of the pattern recognition process was then examined in relation to those who reported having a new major stroke; AUC, risk ratio, sensitivity, specificity, as well as positive and negative predictive values were reported.

Survival modeling was utilized to determine the predictive value of age and gender on the risk of incident stroke determined using diagnosis versus pattern recognition protocols. Due to the high likelihood of ties in repeated measures using survey data, the exact marginallikelihood method was utilized to handle ties. The proportional hazard assumption was tested using Schoenfeld residuals.

Longitudinal modeling was utilized to examine rates of change in episodic memory preceding and following the onset of different types of stroke determination methods. Longitudinal modeling was utilized because it reliably estimates both level and rate of change in the outcome while adjusting for time-invariant and time-varying covariates [11]. Random intercepts were utilized to identify time-invariant capability; random slopes were utilized to model individual differences in the rate of change in cognition over time. An association between time and baseline capability was utilized to control for regression to the mean [12]. Covariate adjustment included age in years and female sex. Nested models were utilized to examine modeling assumptions. Models began by incorporating rates of change in episodic memory among those experiencing pattern-detected (p-)stroke (model 1), then for those reporting major stroke (model 2), then either experiencing p-stroke or diagnosed major stroke (model 3), and finally incorporating the overlap between incident p-stroke and major stroke (model 4). 


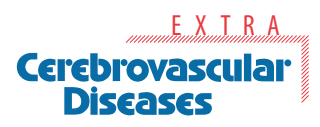

Table 2. Concordance between diagnoses of major stroke and incident cerebrovascular and ischemic diseases detected using a longitudinal pattern recognition methodology

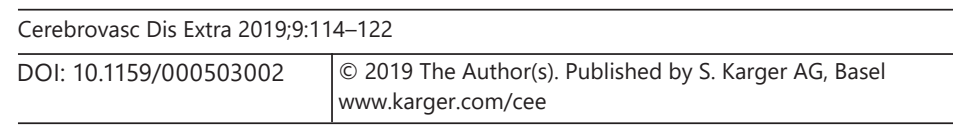

\begin{tabular}{lrl}
\hline & Major stroke & No major stroke \\
\hline p-stroke & 1,365 & 7,499 \\
No p-stroke & 656 & 6,593 \\
AUC (95\% CI) & 0.580 & $(0.569-0.591)$ \\
Risk ratio (95\% CI) & 1.78 & $(1.634-1.939)$ \\
Sensitivity & & 0.675 \\
Specificity & & 0.468 \\
Positive predictive value & & 0.154 \\
Negative predictive value & & 0.910 \\
\hline
\end{tabular}

See text for abbreviations.

\section{Results}

Sample descriptive statistics (Table 1) revealed that respondents were on average in their 60s, and the majority were female. On average, more than half of respondents $(50.54 \%)$ were identified as having $\mathrm{p}$-stroke-related decline over the observation period; $\mathrm{p}$-stroke occurred $4.63(\mathrm{SD}=4.72)$ years after the start of observation when detected using episodic memory. Among those with p-stroke, diagnoses of major stroke were reported 9.59 (SD = $4.74)$ years following the start of observation reflecting a difference of 4.963 (4.650-5.275) years $(p<0.001)$.

The p-stroke IR using episodic memory was 41.48/1,000 (95\% CI $=40.60-42.37)$ personyears. The IR of diagnosed major stroke was much lower at 8.13/1,000 (95\% CI = 7.77-8.51) person-years. Concordance between major stroke and p-stroke was reasonable, highlighting a large burden of cases that remain undiagnosed (Table 2).

Efforts to utilize both episodic memory and orientation in a subset of respondents (online suppl.Table S1; for all online suppl. material, see www.karger.com/doi/10.1159/000503002) revealed that orientation-detected p-strokes were more common in those with episodicmemory-detected p-stroke, and each was fairly common both on its own and in conjunction with the second p-stroke identification. Notably, while detection of p-stroke from both episodic and orientation measures was the most sensitive (sensitivity $=0.81$, specificity $=$ 0.32 ), detection of both episodic memory and orientation-related strokes was a more specific indicator (sensitivity $=0.42$, specificity $=0.74$ ).

Analyses examining demographic correlates of p-stroke (online suppl. Table S2) revealed that age was associated with an increased incidence of p-stroke and major stroke. Sensitivity analyses utilizing c-log-log modeling to determine the impact of categorical time-to-event data provided results similar to those shown in the survival analyses above (online suppl. Table S3).

To examine differences in the severity of cognitive effects between diagnostic and p-stroke identification protocols, longitudinal analyses estimated the size of stroke-related decreases in cognitive function both for stroke and for p-stroke independently and in relation to one another (online suppl. Table S4). Analyses identified changes in cognitive function that were approximately 2 words lower (58.8\% of one standard deviation for episodic memory) after a p-stroke (model 1). Figure 2 shows results from this model showing simply the rate of cognitive decay expected in respondents with p-stroke and those without. Interestingly, the rate of decline in episodic memory was smaller among those who reported strokes than those with p-stroke (model 2). Adjusting for both stroke and p-stroke identified independent effects for each and marginally reduced the overall association between major stroke and episodic memory (model 3). Examining the comorbidity (model 4) suggested that p-stroke with 


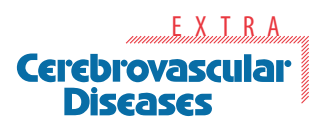

Fig. 2. Differential pathological characterization based on aging pattern in episodic memory. Average curves were calculated using results shown in online supplementary Table S1 (model 1).

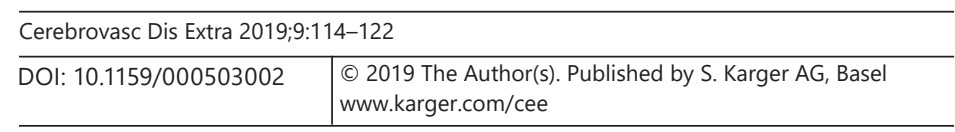

Clouston et al.: Stroke-Like Patterns of Cognitive Decline

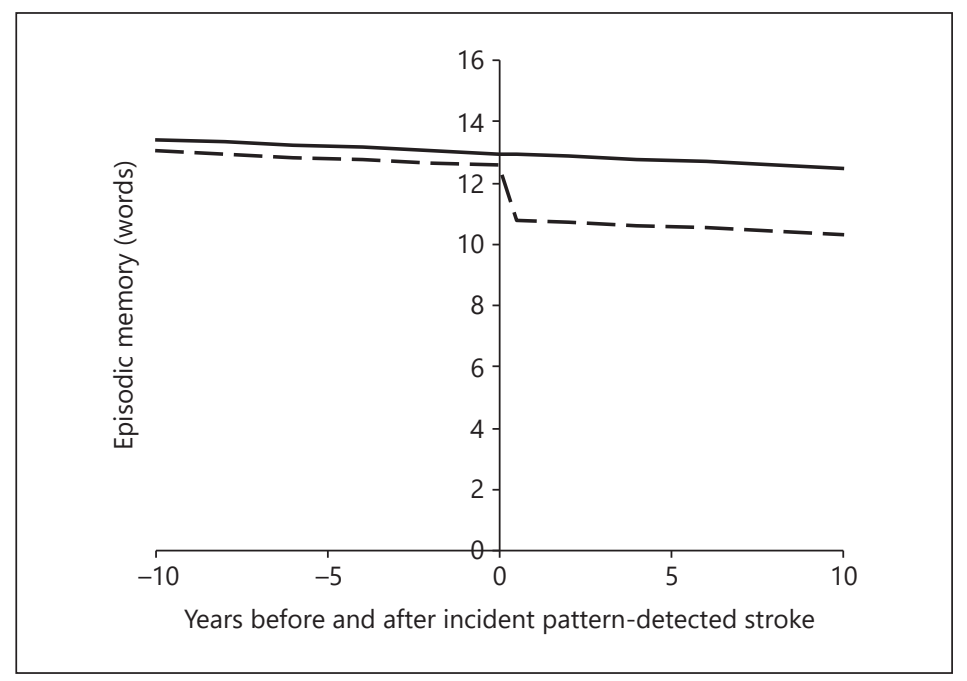

reported stroke had the largest decrement in episodic memory, while p-stroke alone had the second largest decrement, and reported stroke without p-stroke held the smallest association with episodic memory.

\section{Sensitivity Analyses Replicating p-Stroke Analysis Using Orientation}

To examine the potential utility of additional testing to improve identification routines, we examined the utility of the orientation measure, which was collected only in a subgroup of individuals starting at 65 years of age. These results showed that p-stroke occurred approximately at the same time when using orientation $4.90(\mathrm{SD}=4.81)$ and episodic memory 4.63 $(\mathrm{SD}=4.72)$ for detection. The incidence of $\mathrm{p}$-stroke detected using orientation was $20.37 / 1,000$ (19.82-20.93) person-years, approximately half as common as when using orientation as compared to episodic memory (incidence rate ratio $=0.491$ [0.488-0.494], $p<0.001$ ). Survival analyses revealed that age, female sex, education, and Black race were associated with the incidence of orientation-detected p-stroke (online suppl. Table S4).

\section{Discussion}

There is a need to develop new objective indicators for stroke. This study sought, for the first time, to prospectively detect incident strokes using a pattern recognition protocol applied to serial indicators of cognitive functioning. Results showed that there was a potentially high burden of p-stroke in this population, and they also suggested that p-strokes were concordant with incident diagnoses of major stroke. This study examined the shape of longitudinal trajectories in order to identify patterns of cognitive dysfunction consistent with stroke. The method used represents a proof of concept that suggested that we could reliably identify selfreported strokes with fair accuracy. While intriguing, these results are still preliminary and suggest that additional refinement is required to make these types of analyses more useful in identifying stroke.

The proposed method reliably detected major stroke at a much earlier time point than diagnosis, but identified a large number of individuals lacking any diagnosis of major stroke during the observational period. Strokes may range in size and impact, and thus detected strokes may reflect, either, noise or an otherwise undetected stroke. The prevalence of smaller 
lesions and the type, location, and size needed to cause cognitive damage or impairment is unclear [13]. This lack of clarity represents a challenge to understanding clinical and population diagnoses, since gold standard diagnoses often rely on expensive neuroimaging or else on postmortem evidence [14]. Lacking evidence from neuroimaging, individuals or their representatives are asked to determine the timing of symptom onset in order to make a diagnosis [15]. As a result, only major strokes are diagnosed, most small strokes as well as many moderately large ones with only minor symptoms, or with symptoms that occur while sleeping, are never diagnosed. Since these processes are difficult to disentangle from processes like normal aging, studies do not make a clear distinction between causes [6].

A lack of objective and consistent identification methods can result in a lack of sufficient evidence linking specific risk factors to a unique disease etiology [16]. Additionally, substantial bias may arise from studies that examine risk factors specifically for stroke because those identified as experiencing stroke may be a different subset of individuals for whom symptoms were more noticeable or occurred in a context that magnified their impact. Improving our capacity to complete early diagnosis creates an opportunity to lower patient risks, prevent complications, and also help individuals to better prepare for their future [17]. Thus, there is a need to develop methods to reliably identify causes of cognitive decline and to accurately identify methods for reliably diagnosing the etiology of that decay [18]. Years of preclinical cognitive decline in longitudinal data fit the natural history of p-stroke and in turn imply the possibility of utilizing population-based data mining techniques to improve identification. Distinct from models based on neuropsychological test cutoffs and using slope methods, the current method emphasizes patterns of within-person changes over time.

Among respondents who completed both episodic memory and orientation tasks, many of those experiencing p-stroke cognitive decays also showed reduced orientation. The specificity to detect major stroke diagnoses improved when relying on both episodic memory and orientation measures, supporting the view that major stroke is better detectable because it has more severe symptoms. Future work should determine the extent to which the degree of concordance across measures might be used as a grading scheme for stroke severity.

The methods and results of this study suggest that strokes may be characterized by cognitive declines that do not revert. The mechanism for such a pattern is not addressed in the current investigation; however, it likely includes changes in white matter connectivity [19], which result in immediate function impairments in nearby cortical tissue [20]. However, alternative mechanisms may include the activation of glial cells with concurrent cognitive decay [21] and interference in functional pathways necessary for complex learning and memory processes [22].

\section{Limitations}

This study may be one of the earliest studies to specifically focus on modeling both healthy aging while differentiating causes of neuropathology using pattern recognition. Nevertheless, this method is designed to identify p-stroke, rather than to provide definitive diagnoses, so it is impossible to clarify the etiology of p-stroke in this study. This study was limited in relying on self-reported diagnoses of major stroke, which are known to underestimate the actual prevalence of microstrokes and other possible types of cerebrovascular diseases. While this study may have contributed to identifying a large burden of smaller strokes that have implications for cognitive functioning, further research is needed to validate p-stroke detection routines using biomarkers including, for example, neuroimaging markers. Although episodic memory is recognized as a central symptom for neuropathological injury and to measure brain aging, strokes not fitting the pattern utilized for recognition efforts may be missed.

We did not utilize missing data to inform pattern detection routines; however, there is a chance that data are missing due to an incipient stroke. Since such efforts were beyond the 
scope of this study, future work is needed to determine effective methods to integrate informative missing data into these analyses. Respondents in the current study included a wide array of American residents from disparate social and racial backgrounds. Nevertheless, further work is needed in racial/ethnic subgroups to determine whether the efficacy of p-stroke detection efforts is modified by demographic characteristics. Finally, this study had data requirements including a large number of follow-ups occurring over a relatively long period that may make the method less useful in other longitudinal studies of cognitive function in old age.

\section{Implications}

Accurately identifying the burden of stroke in epidemiological studies is expensive and difficult, often relying on neuroimaging in order to identify these factors, thereby resulting in a large burden of disease that remains undiscovered [23]. This is problematic for research since those lacking diagnoses are likely to differ systematically from those who are diagnosed, including due to the level of health care access, and the level of connection with family members or friends who may help to identify symptoms. This study sought to inferentially identify the incidence of stroke by utilizing longitudinal patterns of decline in episodic memory and, in sensitivity analyses, in orientation. These results were promising and highlighted a potentially large burden of heretofore unobserved cerebrovascular diseases in most older Americans. While benefits to research are clear, clinical benefits may include the potential for earlier stroke detection in clinics where cognitive screening is routine. Given the push by the Alzheimer's Association to include cognitive screening in annual wellness visits for US Medicare patients [24], this protocol has the potential to assist clinicians in the earlier detection of stroke in a large proportion of older Americans at a point when interventions may be feasible to reduce the risk of additional strokes. Given the large IR differences, this study suggests that more work is warranted to replicate, extend, and validate the current model using data with more pathological measures.

\section{Acknowledgments}

The HRS is sponsored by the National Institute on Aging (grant No. NIA U01AG009740) and is conducted by the University of Michigan. The authors would like to acknowledge the Integrative Analysis of Longitudinal Studies on Aging (NIH/NIA P01 AG043362) network for supporting the development of this work and the National Institute on Aging for supporting this analysis in particular (NIH/NIA R01 AG058595).

\section{Statement of Ethics}

This secondary analysis of publicly available de-identified data was determined to be not human subject research by the institutional ethics committee.

\section{Disclosure Statement}

The authors have no conflicts of interest, financial or otherwise, to disclose. 
Clouston et al.: Stroke-Like Patterns of Cognitive Decline

\section{References}

1 Yang Q, Tong X, Schieb L, Vaughan A, Gillespie C, Wiltz JL, et al. Vital signs: recent trends in stroke death rates - United States, 2000-2015. MMWR Morb Mortal Wkly Rep. 2017 Sep;66(35):933-9.

2 Gorelick PB, Scuteri A, Black SE, Decarli C, Greenberg SM, Iadecola C, et al.; American Heart Association Stroke Council, Council on Epidemiology and Prevention, Council on Cardiovascular Nursing, Council on Cardiovascular Radiology and Intervention, and Council on Cardiovascular Surgery and Anesthesia. Vascular contributions to cognitive impairment and dementia: a statement for healthcare professionals from the American Heart Association/American Stroke Association. Stroke. 2011 Sep;42(9):2672-713.

3 Schneider BC, Gross AL, Bangen KJ, Skinner JC, Benitez A, Glymour MM, et al. Association of vascular risk factors with cognition in a multiethnic sample. J Gerontol B Psychol Sci Soc Sci. 2015 Jul;70(4):532-44.

4 Howard VJ, McClure LA, Meschia JF, Pulley L, Orr SC, Friday GH. High prevalence of stroke symptoms among persons without a diagnosis of stroke or transient ischemic attack in a general population: the REasons for Geographic And Racial Differences in Stroke (REGARDS) study. Arch Intern Med. 2006 Oct;166(18):1952-8.

5 Ince PG, Minett T, Forster G, Brayne C, Wharton SB, Medical Research Council Cognitive Function and Ageing Neuropathology Study. Microinfarcts in an older population-representative brain donor cohort (MRC CFAS): Prevalence, relation to dementia and mobility, and implications for the evaluation of cerebral small vessel disease. Neuropathol Appl Neurobiol. 2017 Aug;43(5):409-18.

6 Clouston SA, Glymour M, Terrera GM. Educational inequalities in aging-related declines in fluid cognition and the onset of cognitive pathology. Alzheimers Dement (Amst). 2015 Sep;1(3):303-10.

7 Korczyn AD, Vakhapova V, Grinberg LT. Vascular dementia. J Neurol Sci. 2012 Nov;322(1-2):2-10.

8 National Institute on Aging (U01 AG 009740). Health and Retirement Study (HRS). Ann Arbor, MI: University of Michigan; 2014.

9 Baddeley A. Working memory. Science. 1992 Jan;255(5044):556-9.

10 Okura Y, Urban LH, Mahoney DW, Jacobsen SJ, Rodeheffer RJ. Agreement between self-report questionnaires and medical record data was substantial for diabetes, hypertension, myocardial infarction and stroke but not for heart failure. J Clin Epidemiol. 2004 Oct;57(10):1096-103.

11 Rabe-Hesketh S, Skrondal A. Multilevel and longitudinal modeling using Stata. College Station, TX: STATA Press; 2008.

12 Liu M, Qian M, Cheng Q, Berger KI, Shao Y, Turetz M, et al. Longitudinal spirometry among patients in a treatment program for community members with World Trade Center-related illness. J Occup Environ Med. 2012 Oct;54(10):1208-13.

13 Vitali P, Migliaccio R, Agosta F, Rosen HJ, Geschwind MD. Neuroimaging in dementia. Semin Neurol. 2008 Sep; 28(4):467-83.

14 Scheltens P, Blennow K, Breteler MM, de Strooper B, Frisoni GB, Salloway S, et al. Alzheimer's disease. Lancet. 2016 Jul;388(10043):505-17.

15 Pohjasvaara T, Mäntylä R, Ylikoski R, Kaste M, Erkinjuntti T. Comparison of different clinical criteria (DSM-III, ADDTC, ICD-10, NINDS-AIREN, DSM-IV) for the diagnosis of vascular dementia. National Institute of Neurological Disorders and Stroke-Association Internationale pour la Recherche et l'Enseignement en Neurosciences. Stroke. 2000 Dec;31(12):2952-7.

16 Jellinger KA. The enigma of vascular cognitive disorder and vascular dementia. Acta Neuropathol. 2007 Apr; 113(4):349-88.

17 Doubal FN, Ali M, Batty GD, Charidimou A, Eriksdotter M, Hofmann-Apitius M, et al. Big data and data repurposing - using existing data to answer new questions in vascular dementia research. BMC Neurol. 2017 Apr; 17(1):72.

18 Albert MS, DeKosky ST, Dickson D, Dubois B, Feldman HH, Fox NC, et al. The diagnosis of mild cognitive impairment due to Alzheimer's disease: recommendations from the National Institute on Aging-Alzheimer's Association workgroups on diagnostic guidelines for Alzheimer's disease. Alzheimers Dement. 2011 May; $7(3): 270-9$.

19 Chen SQ, Kang Z, Hu XQ, Hu B, Zou Y. Diffusion tensor imaging of the brain in patients with Alzheimer's disease and cerebrovascular lesions. J Zhejiang Univ Sci B. 2007 Apr;8(4):242-7.

20 Agosta F, Pievani M, Sala S, Geroldi C, Galluzzi S, Frisoni GB, et al. White matter damage in Alzheimer disease and its relationship to gray matter atrophy. Radiology. 2011 Mar;258(3):853-63.

21 Bilbo SD, Smith SH, Schwarz JM. A lifespan approach to neuroinflammatory and cognitive disorders: a critical role for glia. J Neuroimmune Pharmacol. 2012 Mar;7(1):24-41.

22 Dacosta-Aguayo R, Graña M, Savio A, Fernández-Andújar M, Millán M, López-Cancio E, et al. Prognostic value of changes in resting-state functional connectivity patterns in cognitive recovery after stroke: A 3T fMRI pilot study. Hum Brain Mapp. 2014 Aug;35(8):3819-31.

23 Amjad H, Roth DL, Sheehan OC, Lyketsos CG, Wolff JL, Samus QM. Underdiagnosis of Dementia: an Observational Study of Patterns in Diagnosis and Awareness in US Older Adults. J Gen Intern Med. 2018 Jul;33(7): 1131-8.

24 Cordell CB, Borson S, Boustani M, Chodosh J, Reuben D, Verghese J, et al.; Medicare Detection of Cognitive Impairment Workgroup. Alzheimer's Association recommendations for operationalizing the detection of cognitive impairment during the Medicare Annual Wellness Visit in a primary care setting. Alzheimers Dement. 2013 Mar;9(2):141-50. 